\section{MR-Venography Using High Resolution True-FISP}

\begin{abstract}
Summary. A new fast MR-venography approach using a high resolution True-FISP imaging sequence was investigated in $\mathbf{2 0}$ patients suffering from 23 deep vein thromboses. Diagnosis was proven by $\mathrm{x}$-ray venography, $\mathrm{CT}$ or ultrasound examination. The presented technique allowed for clear thrombus visualization with a high contrast to the surrounding blood pool even in calf veins. Acquisition time was less than $\mathbf{1 0}$ minutes for imaging the pelvis and the legs. No contrast media was needed. The presented high resolution True-FISP MR-venography is a promising non-invasive, fast MR-venography approach for detection of deep venous thrombosis.
\end{abstract}

Key words: MR-venography - True-FISP - Contrast enhancement

Hochaufgelöste True-FISP MR-Phlebographie. Eine neue, schnelle, örtlich hochaufgelöste MR-Phlebographietechnik mit einer axialen True-FISP Bildgebungssequenz wurde an $20 \mathrm{~Pa}-$ tienten mit 23 nach-gewiesenen tiefen Beinvenenthrombosen untersucht. Die Befunde wurden mit einer konventionellen Röntgenphlebographie, einer CT oder einer Sonographie gesichert. Die vorgestellte Technik erlaubte in allen Fällen eine Thrombusdarstellung mit hohem Kontrast zum umgebenden venösen Blut, wobei aufgrund der hohen Ortsauflösung auch die Unterschenkelvenen beurteilt werden konnten. Die Datenaufnahmezeit zur Untersuchung des Beckens und der Beine betrug weniger als 10 Minuten. Kontrastmittel wurde nicht benötigt. Die vorgestellte MR-Phlebographietechnik unter Verwendung einer örtlich hochauf-gelösten True-FISP Sequenz ist eine neue, vielversprechende, nicht-invasive Technik zur Diagnostik der tiefen Bein- und Beckenvenenthrombose.

Schlüsselwörter: MR-Phlebographie - True-FISP - Kontrastverstärkung

\section{Introduction}

Over the last couple of years developments of magnetic resonance imaging (MRI) hardware and software enabled the acquisition of high-quality images using fast imaging with steady-state precession (True-FISP) [1-3]. With this tech-

Fortschr Röntgenstr 2001; 173: 686-690

(c) Georg Thieme Verlag Stuttgart · New York

ISSN $1438-9029$
E. Spuentrup ${ }^{1,2}$, A. Buecker ${ }^{1}$, M. Stuber ${ }^{2,3}$, R. W. Günther ${ }^{1}$

${ }^{1}$ Department of Diagnostic Radiology, Aachen University of Technology, Germany

2 Department of Medicine (Cardiovascular Division), Beth Israel Deaconess Medical Center and Harvard Medical School, Boston, MA

${ }^{3}$ Philips Medical Systems, Best, NL

nique, a high contrast between blood (hyperintense) and muscle (hypointense, i.e. myocardium) has been reported. Due to the intrinsic T1/T2 contrast in True-FISP $[2,4,5]$, neither exogenous contrast agents nor pre-pulses are needed. Optimal image quality with reduced artifacts in True-FISP sequences can be achieved using a minimally short repetition time (TR) during the steady-state of the signal $[1,3]$. In addition to this, the intrinsic flow compensation of True-FISP in all three spatial coordinates allows for suppression of artifacts originating from constant flow [5]. Utilizing increased gradient performance as now available on clinical MR-scanners, short TR and TE became feasible even for high resolution True-FISP sequences. The high spatial resolution in conjunction with the intrinsic high signal of blood independent of flow makes True-FISP a potentially favorable imaging sequence for MR-venography. Furthermore, fast data acquisition in True-FISP sequences might allow for axial slice orientations with improved delineation of thrombus head configuration in sufficiently short acquisition times.

\section{Methods}

High resolution True-FISP-venography was implemented on a commercial 1.5 T Gyroscan ACS-NT whole body MR system (Philips Medical Systems, Best, NL) equipped with cardiac software (INCA2) and a commercially available PowerTrak $^{\circledR} 6000$ gradient system $(23 \mathrm{mT} / \mathrm{m}, 219 \mu$ s rise time). For signal acquisition, a commercial 4-element body synergy coil was positioned at the level of the pelvis and vena cava inferior. Signal from the lower extremities was obtained using a second (5-element) synergy coil. Using both coils and a floating table with a step by step technique, data were acquired from the whole region of interest (crus to infra-renal vena cava) without repositioning of the patient. All subjects were examined in the supine position.

\section{Scout scanning}

For localization of the venous blood stream, a multi stack 2D True-FISP scout scan was used (TE $1.2 \mathrm{~ms}, 128 \times 128$ matrix, $440 \mathrm{~mm}$ field-of-view, 27 slices, slice thickness $10 \mathrm{~mm}$, acquisition time $8 \mathrm{sec}$ ).

\section{Imaging sequence for True-FISP MR-venography}

MR-venography was acquired using a 2D True-FISP imaging sequence with a centric ordered $\mathrm{k}$-space acquisition scheme, a TR of $5.0 \mathrm{~ms}$ and a TE of $2.5 \mathrm{~ms}$. A constant excitation angle of $85^{\circ}$ was applied. Because steady state conditions are important for image quality in True-FISP, 20 start-up cycles preceded the 
imaging portion of the sequence. The field-of-view was $440 \mathrm{~mm}$ with a $512 \times 512$ scan matrix, resulting in an in-plane resolution of $860 \times 860 \mu \mathrm{m}^{2}$. The slice thickness was $5 \mathrm{~mm}$. For the pelvis two stacks with 20 slices each were acquired asking the patient to take shallow breaths only to minimize breathing artifacts. MR-venography of the pelvis and the legs was acquired using multiple stacks and a floating table with a step-by-step technique. This ensured that the imaging slab was always in the isocenter of the magnet in order to avoid banding artifacts originating from field inhomogeneities. Data acquisition time was less than $10 \mathrm{~min}$ for a $100 \mathrm{~cm}$ region of interest in craniocaudal orientation.

\section{Subjects}

True-FISP MR-venography was investigated in 40 lower extremities in 20 patients ( $14-88$ years old). 13 patients suffered from left or right side thrombosis, 5 patient from bilateral thrombosis, resulting in 23 thromboses in 40 extremities. Two patients had no thrombosis in the investigated region. The proximal thrombus head was localized in the vena cava $(n=6)$, iliac vein $(n=7)$, superficial/common femoral vein $(n=5)$ or popliteal vein $(n=4)$. X-ray phlebography $(n=7), C T(n=8)$ or ultrasound $(n=5$, contraindication for X-ray contrast agent or pregnancy) was performed for comparison $[6,7]$.

\section{Data analysis}

True-FISP MR-venography was analyzed by two radiologists. The investigators were asked to identify thrombus localization. Subsequently, data were compared with x-ray phlebography, CT or ultrasound examination.

Signal-to-noise ratios of the venous blood pool at the level of the iliac, femoral and popliteal veins and thrombus-blood-pool contrast-to-noise ratios [12] were calculated from regions of interest drawn in the venous blood pool and the thrombus (maximal fitting circle). Noise was defined as the standard deviation in a region of air in frequency encoding direction.

\section{Results}

MR studies were completed in all subjects without complications. Data acquisition time was less than 10 minutes. On all the acquired True-FISP MR-venography images, signal-to-noise ratio and contrast-to-noise ratio could be evaluated. Veins and arteries were successfully differentiated including main calf veins using adjacent slices.

Fig. 1 a shows representative images of a patient without thrombosis. External iliac veins are displayed with a bright signal. In contrast, veins occluded by thrombus demonstrated a signal loss and an enlarged vessel diameter (Fig. 2). The proximal thrombus head of an acute deep venous thrombosis (Fig. 3) is clearly delineated demonstrating a dark signal and a high contrast to the surrounding bright venous blood stream. In all cases the thrombus head could be correctly localized on the True-FISP MR-venography.

Although the aim of this study was to investigate the feasibility of True-FISP MR-venography, in two patients with insufficient ultrasound examination conditions but contraindication to Xray phlebography, True-FISP-venography could demonstrate
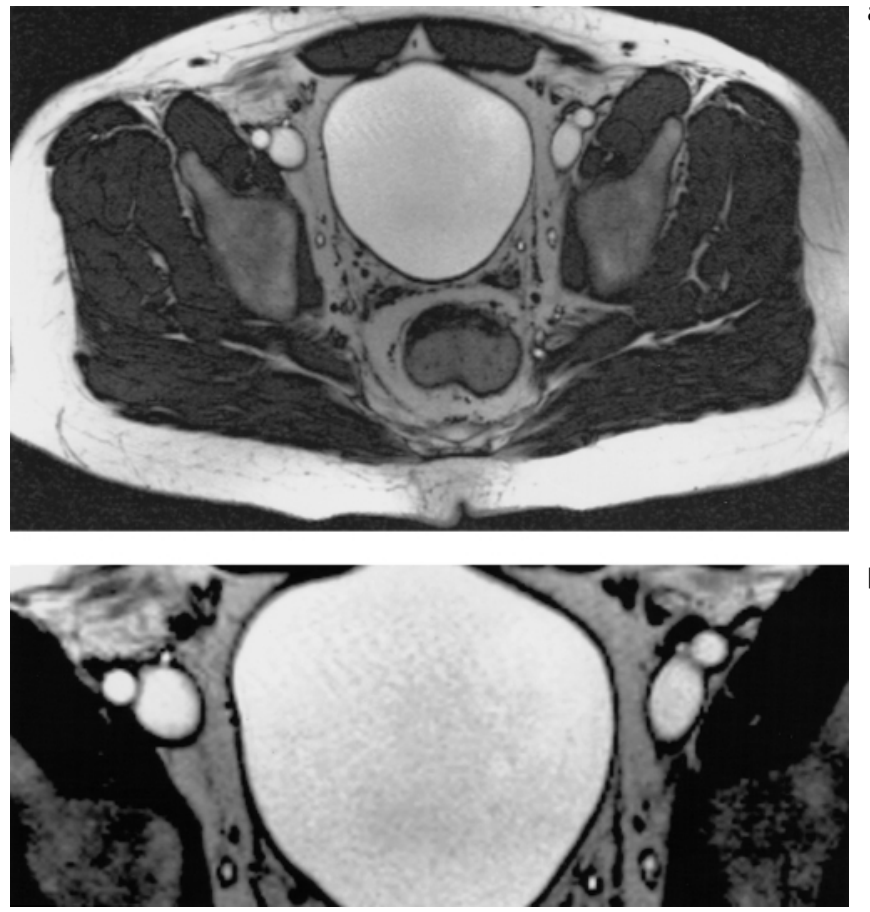

Fig. 1 True-FISP MR-venography (TR $5.0 \mathrm{~ms}$, TE $2.5 \mathrm{~ms}, 860 \times 860 \mu \mathrm{m}$ in-plane resolution) in a 42 year old patient without thrombosis. Veins and arteries are displayed with a bright signal. A black rim surrounding the vessel lumen is readily apparent (magnification).

important additional findings like common iliac vein or inferior vena cava thrombosis (Fig. 4). In two further cases, unknown contralateral thrombosis was seen with True-FISP MR-venography. Two tumor masses with venous compression syndrome and one vena cava compression due to pregnancy (Fig. 2 c) were found on the True-FISP MR-venography images.

Objective analysis of the pelvic and deep leg veins demonstrated a mean signal-to-noise ratio of the blood-pool of $54 \pm 19$ and a high mean thrombus-blood-pool contrast-to-noise ratio of $46 \pm 16$.

\section{Discussion}

Detection of acute deep vein thrombosis is of crucial importance due to potentially severe complications including acute or chronic pulmonary embolism [8]. X-ray phlebography and ultrasound are well established for diagnosis of deep vein thrombosis. But there are contraindications for X-ray phlebography like pregnancy, hyperthyreosis or renal failure. Furthermore, contrast venography is semi-invasive and may fail to depict some segments of the venous system in up to $10-20 \%$ [7]. Ultrasound venography has been established as a noninvasive tool for deep vein thrombosis detection with the limitation of low sensitivity for iliac veins, vena cava inferior or calf veins thromboses. Furthermore, ultrasound is examiner dependent and time-consuming. Thus, a non-invasive MRtechnique for fast visualization of the venous system may be a useful clinical tool. To obtain sufficient visualization of small veins or thrombi in MR imaging, high spatial resolution is needed with a high contrast-to-noise ratio. Therefore, we sought to investigate the potential of a fast high resolution 


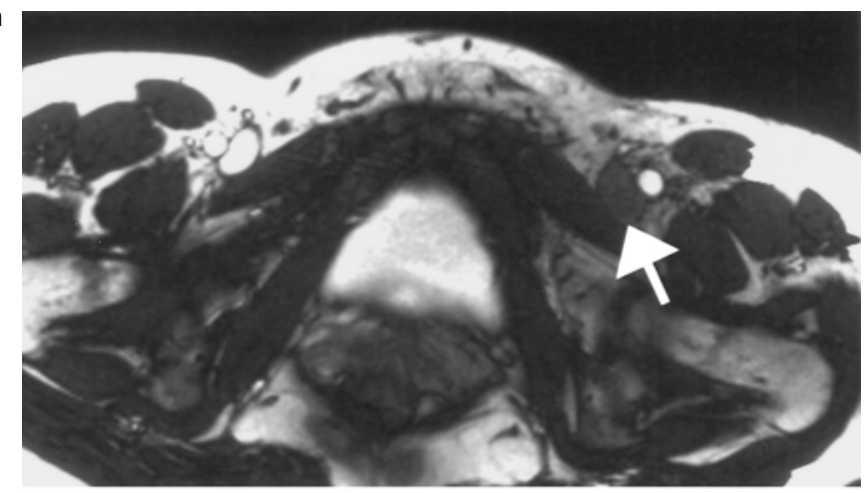

b

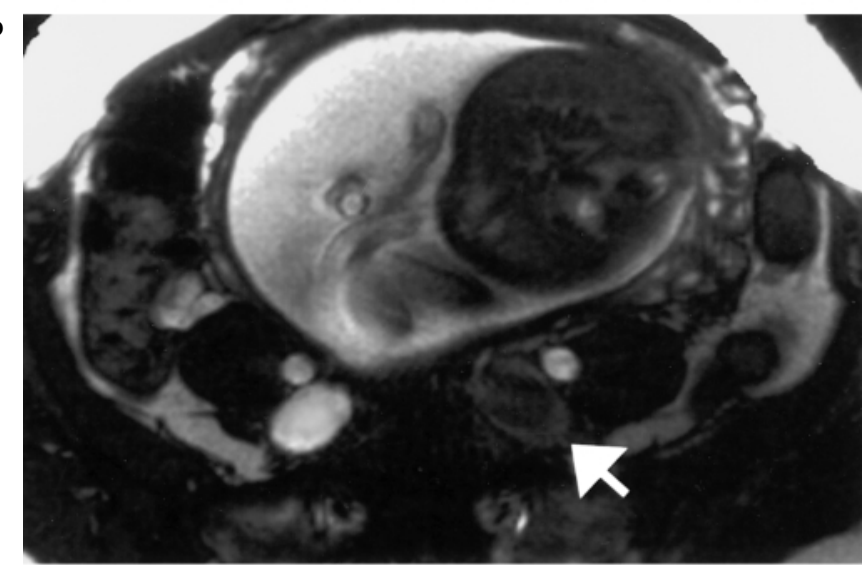

C

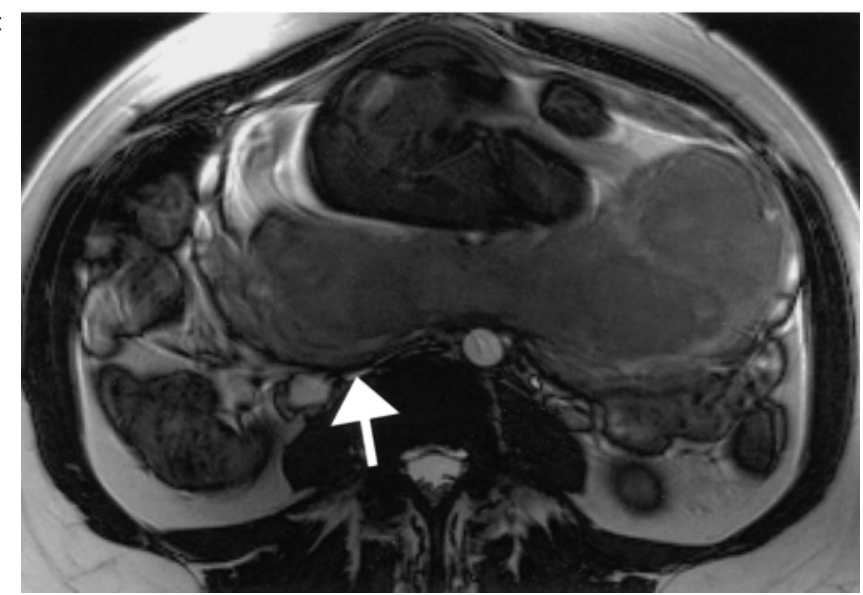

Fig. 2 True-FISP MR-venography (TR $5.0 \mathrm{~ms}$, TE $2.5 \mathrm{~ms}, 860 \times 860 \mu \mathrm{m}$ in-plane resolution) in a 31 year old pregnant patient. Complete left femoral (a) and iliac (b) vein thrombosis is diagnosed because of the dark signal, whereas open superficial and deep right veins show a bright signal. The inferior vena cava is highly compressed due to pregnancy (arrow in c).

True-FISP sequence for MR-venography, which may allow for a high-contrast MR-venography without costly contrast agent.

\section{Technical considerations of MR-venography using high resolution True-FISP}

True-FISP sequences demonstrate a T1/T2 contrast due to data acquisition in a steady state condition of the transverse magnetization [9]. Therefore, the long T2 of blood allows for high signal intensities of the blood pool. These signal properties are well known from functional cardiac studies $[1,3,9]$. However, in such lower resolution cardiac images, fat is nicely suppressed if a low number of excitations and centric k-space sampling is used [10]. In contrast to this, in our high resolution True-FISP approach with a $512 \times 512$ matrix, enhanced excitations are used which might explain the bright signal of fat in the presented True-FISP MR-venography images. The lack of fat suppression in our sequence did not limit vessel depiction, because a typical black rim surrounding the vessels allowed for sharp delineation of the vessel lumen even in small veins (Fig. 1). This black rim is probably due to the echo time of $2.5 \mathrm{~ms}$ yielding a near out-of-phase situation for fat and water protons [9]. In our series, all thrombus demonstrated a hypointense signal when compared to the venous blood pool, which may be explained by the relatively short $\mathrm{T} 2$ of clots [11]. However, further investigations including clots of various ages are needed to fully understand the contrast in MR-venography using TrueFISP sequences.

Due to the intrinsic flow-compensation of True-FISP in all three spatial coordinates, flow-artifacts are totally suppressed if slow constant flow is present [5]. In contrast to True-FISP MRangiography, which requires data acquisition during diastole to avoid artifacts from systolic aortic pulse [12], imaging of slow more constant venous blood may be possible without k-space segmentation and therefore with more stable steady-state conditions. With the presented True-FISP imaging sequence, the venous blood stream of the lower extremities including the pelvic veins and infrarenal vena cava could be visualized without motion artifacts. However, the impact of venous flow on True-FISP images remains to be investigated.

\section{Clinical considerations of MR-venography using high resolution True-FISP}

In our series, True-FISP MR-venography allowed for clear visualization of the thrombus in all cases even in the main veins of the crus (Fig. $3 \mathbf{a}, \mathbf{b}$ ). The presented technique is a fast ( $<10 \mathrm{~min}$ ) imaging tool for routine diagnosis of deep vein thrombosis, even with transaxial slice orientation and high spatial resolution. First MR-venography techniques have used pedal contrast media administration and coronal $3 \mathrm{D}$ gradientecho sequences as known from MR-angiography, resulting in excellent MR-venograms [13]. However, this approach is still semi-invasive, needs costly contrast agents and acquires coronal slices. Axial imaging planes and high spatial resolution as used in the presented approach may have the potential of better visualization of thrombus head configuration with respect to the surrounding blood pool and contact to the vessel wall when compared to coronal orientations due to lesser partial volume effect in perpendicular to the vessel orientations. Although axial slice orientation requires much more images than coronal slice orientation in order to cover the same field-of-view, with the presented fast imaging True-FISP MRvenography approach, limitations due to long acquisition times for large examined regions of interest are no longer present.

In our first True-FISP MR venography study using True-FISP imaging, 23 of 23 deep venous thromboses were successfully visualized. Furthermore, in this first feasibility study in two cases unsuspected bilateral thrombosis were found on TrueFISP MR-venography. In one further case, True-FISP MRvenography could clearly show a large floating thrombus head 
a, b
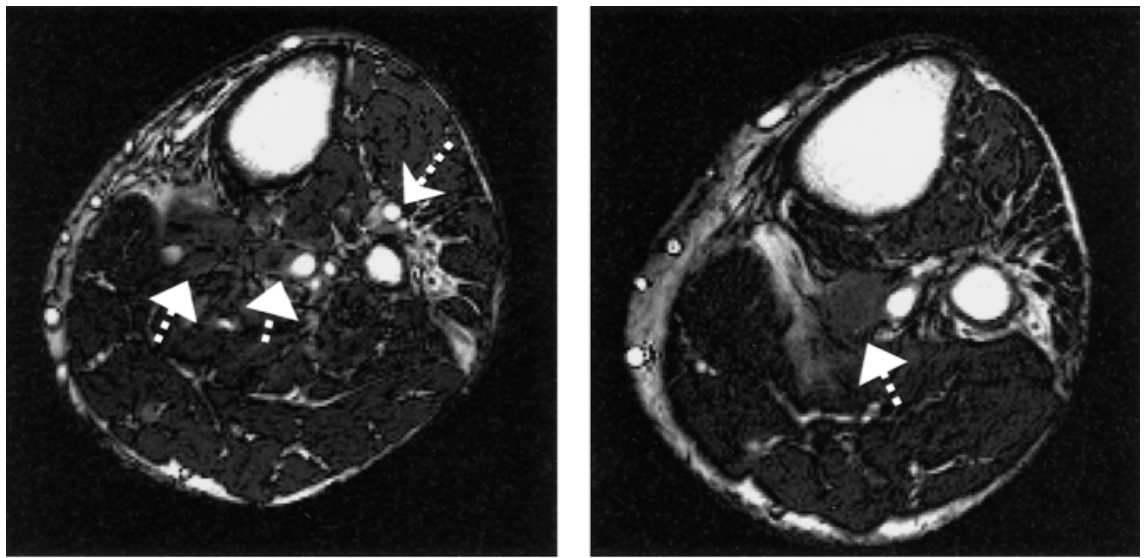

c, d
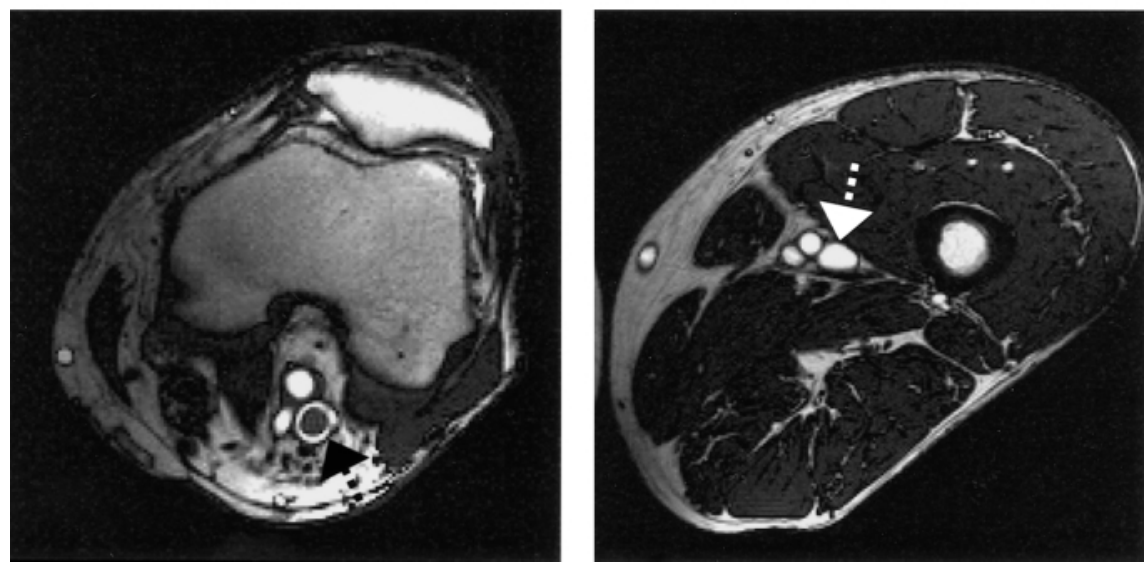

$\mathrm{a}, \mathrm{b}$

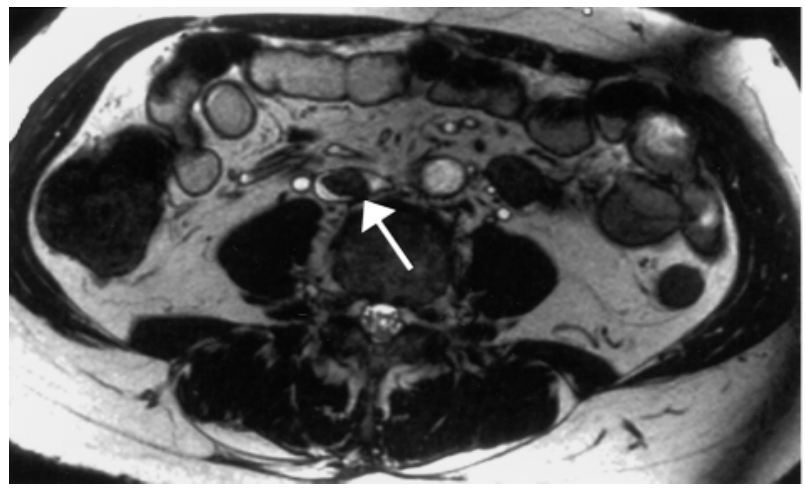

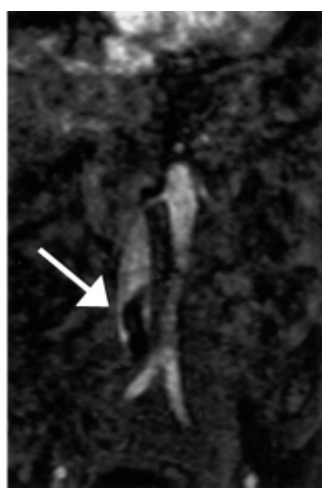

Fig. 3 True-FISP MR-venography (TR $5.0 \mathrm{~ms}$, TE $2.5 \mathrm{~ms}, 860 \times 860 \mu \mathrm{m}$ in-plane resolution) in a 75 year old male patient with acute lower extremity thrombosis. Slices at the level of proximal calf (a), trifurcation (b), popliteal vein (c) and distal femoral vein (d). A low signal free floating thrombus head in the popliteal vein is seen, whereas the femoral vein is of normal (bright) signal intensity. Thrombosis of the calf veins and trifurcation is depicted by enlarged low signal vessel lumen. Only the anterior group shows normal signal.

Fig. 478 year old female patient with femoral thrombosis as proven by ultrasound examination. Due to meteorism, iliac veins and inferior vena cava could not be sufficiently examined by ultrasound. X-ray phlebography was not performed because of hyperthyroidism. True-FISP MR-venography clearly shows inferior vena cava thrombosis (a) as proven by additionally performed contrast enhanced MR-imaging (b). in the inferior vena cava (Fig. 4), a highly important finding for therapy. In two other patients, tumor masses causing venous compression were diagnosed on the MR-images. This shows the potential of MRI for examination of both legs and the pelvis including the soft tissue, which is automatically performed with our transaxial True-FISP MR-venography approach.

MR-venography might be the technique of choice in case of pregnancy or for patients with contraindication to contrast media administration. In pregnancy, initial clots are often located in the iliac veins. Exclusion of vena cava inferior thrombosis, which is essential for therapy strategy often is not achievable by ultrasound. Due to potential vena cava compression in pregnant women and in order to minimize patient discomfort, a fast MR-venography is needed, which can be easily performed with True-FISP MR-venography (Fig. 2).
Recently, new MR-approaches have been introduced allowing for fast detection of pulmonary embolism $[14,15]$. Combined with fast MR-venography and without the need for additional contrast agent application, a complete diagnosis can be achieved in one session and with only minor prolongation of measurement time. The presented True-FISP MR-venography seems to be a powerful and efficient tool for that purpose.

Although correct diagnosis of deep vein thrombosis was successfully established in this first feasibility study, there are some limitations of this technique. In the presented True-FISP MR-venography, arteries and veins are displayed with a bright signal, which makes MIP reconstruction not useful. However, the bright arteries may be used as landmarks, especially if veins are occluded. For validation of the clinical usefulness of this new technique, further studies involving more patients and 
only one gold standard for comparison are needed. Furthermore, the superiority of True-FISP MR-venography when compared to other non-invasive tools like ultrasound remains to be investigated.

\section{Conclusions}

The presented MR-venography using high resolution True-FISP allows for fast high-resolution and high-contrast visualization of deep vein thrombosis.

\section{References}

${ }^{1}$ Carr J, Simonetti O, Kroeker R, Bundy J, Pereles S, Finn JP. Segmented True FISP - An Improved Technique for Cine MR Angiography. Proceedings of the International Society for Magnetic Resonance in Medicine (abstract) 2000; 1: 199

2 Oppelt A, Graumann R, Barfuss H, Fischer H, Hartl W, Shajor W. FISP - a new fast MRI sequence. Electromedica 1986; 54: 15-18

${ }^{3}$ Heid O. True FISP Cardiac Fluoroscopy. Proceedings of the International Society for Magnetic Resonance in Medicine (abstract) 1997; 1: 320

${ }^{4}$ Duerk JL, Lewin JS, Wendt M, Petersilge C. Remember true FISP? A high SNR, near 1-second imaging method for T2-like contrast in interventional MRI at 2 T. J Magn Reson Imaging 1998; 8: 203 208

${ }^{5}$ Haacke EM, Wielopolski PA, Tkach JA, Modic MT. Steady-state free precession imaging in the presence of motion: application for improved visualization of the cerebrospinal fluid. Radiology 1990; 175: $545-552$

${ }^{6}$ Loud PA, Grossman ZD, Klippenstein DL, Ray CE. Combined CT venography and pulmonary angiography: a new diagnostic technique for suspected thromboembolic disease. AJR Am J Roentgenol 1998; 170: 951 -954

${ }^{7}$ Fraser JD, Anderson DR. Deep venous thrombosis: recent advances and optimal investigation with US. Radiology 1999; 211: 9-24

${ }^{8}$ Maki DD, Kumar N, Nguyen B, Langer JE, Miller WT, Gefter WB. Distribution of thrombi in acute lower extremity deep venous thrombosis: implications for sonography and CT and MR venography. AJR Am J Roentgenol 2000; 175: 1299-1301

${ }^{9}$ Haacke EM, Tkach JA. Fast MR imaging: techniques and clinical applications. AJR Am J Roentgenol 1990; 155: 951 - 964

${ }^{10}$ Deimling $\mathrm{M}$, Heid O. True FISP Imaging with Inherent Fat Cancelation. Proc Intern Soc Magn Reson Med (abstract) 2000; 1: 1500

${ }^{11}$ Rapoport S, Sonstman HD, Pope C, Camputaro CM, Holcomb W, Gore JC. Venous clots: evaluation with MR imaging. Radiology 1987; $162: 527-530$

12 Spuentrup E, Manning WJ, Boernert P, Kissinger KV, Stuber M. Navigator-gated free-breathing 3D True-FISP projection MRA of the renal arteries with 2D pencil aortic spin labeling pulse. Proc Intl Soc Mag Reson Med 2001; 9: 306

${ }^{13}$ Ruehm SG, Zimny K, Debatin JF. Direct contrast-enhanced 3D MR venography. Eur Radiol 2001; 11: 102-112

${ }^{14}$ Haage P, Bucker A, Kruger S, Adam G, Glowinski A, Schaffter T, Weiss S, van Vaals JJ, Gunther RW. [Radial k-scanning for real-time MR imaging of central and peripheral pulmonary vasculature]. Röfo Fortschr Geb Röntgenstr Neuen Bildgeb Verfahr 2000; 172: $203-206$

${ }^{15}$ Kauczor HU. Contrast-enhanced magnetic resonance angiography of the pulmonary vasculature. A review. Invest Radiol 1998; 33: $606-617$
Elmar Spuentrup, MD

Department of Diagnostic Radiology

University Hospital, Technical University of Aachen

Pauwelsstraße 30

52057 Aachen

Tel. 0049-241-8088332

Fax 0049-241-8888499

E-mail: spuenti@rad.rwth-aachen.de 